\title{
LGR4 and its role in intestinal protection and energy metabolism
}

\author{
Ziru $\mathrm{Li}^{1}$, Weizhen Zhang $^{1,2 *}$ and Michael W. Mulholland ${ }^{1 *}$ \\ ${ }^{1}$ Department of Surgery, University of Michigan Medical Center, Ann Arbor, MI, USA, ${ }^{2}$ Department of Physiology and \\ Pathophysiology, Peking University Health Science Center, Beijing, China
}

Leucine-rich repeat-containing $G$ protein-coupled receptors were identified by the unique nature of their long leucine-rich repeat extracellular domains. Distinct from classical $G$ protein-coupled receptors which act via G proteins, LGR4 functions mainly through $W n t / \beta$-catenin signaling to regulate cell proliferation, differentiation, and adult stem cell homeostasis. LGR4 is widely expressed in tissues ranging from the reproductive system, urinary system, sensory organs, digestive system, and the central nervous system, indicating LGR4 may have multiple functions in development. Here, we focus on the digestive system by reviewing its effects on crypt cells differentiation and stem cells maintenance, which are important for cell regeneration after injury. Through effects on Wnt/ $\beta$-catenin signaling and cell proliferation, LGR4 and its endogenous ligands, Rspondins, are involved in colon tumorigenesis. LGR4 also contributes to regulation of energy metabolism, including food intake, energy expenditure, and lipid metabolism, as well as pancreatic $\beta$-cell proliferation and insulin secretion. This review summarizes the identification of LGR4, its endogenous ligand, ligand-receptor binding and intracellular signaling. Physiological functions include intestinal development and energy metabolism. The potential effects of LGR4 and its ligand in the treatment of inflammatory bowel disease, chemoradiotherapy-induced gut damage, colorectal cancer, and diabetes are also discussed.

Keywords: LGR4, R-spondin, digestive system, energy metabolism, diabetes, colon cancer

\section{Introduction}

Leucine-rich repeat-containing $\mathrm{G}$ protein-coupled receptors (LGRs) are a distinct group of highly conserved proteins of the GPCRs family, characterized by a large extracellular domain (ectodomain) that harbors multiple copies of leucine-rich repeats (LRRs) (1). LRRs represent amphipathic sequences with leucine as the predominant hydrophobic residue and are important for protein-protein interaction (2). The packing of similar repeats allows the formation of a specific hydrogen bond network between neighboring repeats to form a unique tertiary structure (3). LRRs are involved in ligand binding (4), connected via a cysteine-rich region to a seven-transmembrane (TM) domain responsible for heterotrimeric $\mathrm{G}$ protein activation (5).

LGRs are divided into three subgroups (groups A-C) $(1,6)$. Group A receptors include FSH receptor (LGR1), LH receptor (LGR2), and TSH receptor (LGR3), which recognize follicle-stimulating hormone (FSH), luteinizing hormone ( $\mathrm{LH})$, and thyroid-stimulating hormone (TSH), respectively $(7,8)$. These receptors contain seven to nine LRRs in their ectodomains and long hinge regions connecting the LRR domains to TM domains. Group C LGRs have similar number of LRRs but contain a low-density lipoprotein receptor class $\mathrm{A}$ domain motif at the $\mathrm{N}$ terminus and a short

Li Z, Zhang $W$ and Mulholland MW (2015) LGR4 and its role in intestinal protection and energy metabolism. Front. Endocrinol. 6:131. doi: 10.3389/fendo.2015.00131 
hinge region between the LRR domain and the 7TM domain. Group C LGRs include RXFP receptor 1 (LGR7) and RXFP receptor 2 (LGR8), recognizing relaxin and INSL3 (insulin-like peptide $3)$, respectively $(1,7,8)$. The group B receptors include LGR4, LGR5, and LGR6, which are characterized by a long ectodomain containing 17 LRR repeats $(6,8)$ flanked by the N-terminal LRRNT region and the C-terminal LRRCT region (8). Group B receptors play crucial roles in embryonic development and are involved in several types of cancer (9). They have also drawn significant attention recently because of their roles in adult stem cells, especially when LGR5 and LGR6 were identified as stem cells markers in multiple adult tissues (10-12).

The ligands of LGR4-6 remained unidentified for a prolonged time. In 2011, the secreted R-spondin proteins (Rspo1-4) were identified as the endogenous ligands for these receptors to regulate cell proliferation, differentiation, and adult stem cell maintenance through the activation of Wnt signaling pathways $(13,14)$. Details on the binding between R-spondins and LGRs, and the subsequent intracellular signaling pathways are still under investigation. Recent studies have also revealed a relationship between LGR4 and energy metabolism in areas ranging from food intake and obesity to lipid metabolism.

\section{Identification of LGR4}

In 1998, based on the knowledge that large G protein-coupled seven-TM receptors for LH, FSH, and TSH contain LRRs which interact with glycoprotein ligands, and the theory that the putative glycoprotein hormone receptor sequences are conserved in Drosophila and sea anemone $(15,16)$, human sequences related to the sea anemone and Drosophila glycoprotein hormone receptors $(15,16)$ were sought using expression sequence tags. Fragments of two new mammalian receptors in the subfamily of leucinerich repeat-containing $G$ protein-coupled receptors (LGR) were identified. Adding to the three known LGRs, these two new mammalian receptors were named LGR4 and LGR5 (17).

The full-length cDNAs for these novel receptors were isolated using RT-PCR and repeated screening of sub-libraries from rat ovary or human placenta enriched with each receptor cDNA. LGR4 cDNA from rat ovary consists of 3,504 base pairs with a predicted open reading frame (ORF) of 951 amino acids, whereas LGR5 from human placenta has 4,208 base pairs with a 907 amino acids ORF (17). Similar to three known glycoprotein hormone ( $\mathrm{LH}, \mathrm{FSH}$, and TSH) receptors, LGR4 and LGR5 are characterized by multiple LRR sequences. The ectodomains of LGR4 and LGR5 are composed of 17 LRR motifs, each 22-24 amino acids in length (17). In contrast to the restricted tissue expression pattern of known gonadotropin and TSH receptors, these new receptors were found in multiple tissues (17).

Identification of this expanding family of LGRs promoted studies to identify putative ligands and to unravel the evolutionary origin of proteins in this subfamily of receptors.

\section{Ligands of LGR4}

The R-spondin (Rspo) protein family is a group of four secreted proteins (Rspo1-4) that were isolated as strong potentiators of Wnt $/ \beta$-catenin signaling (18-20). These proteins share $40-60 \%$ identity between each other and a similar structure with a cysteine-rich furin-like domain preceding a thrombospondin-like domain $(21,22)$. Despite their similarity, the four known Rspos serve in different developmental events. Rspo1 regulates sexual development; Rspo 2 modulates development of limbs, lungs, and hair follicles; Rspo3 is involved in placenta development; and Rspo4 affects nail development (23).

Beginning in 2011, several groups have demonstrated that Rspondins (Rspo 1-4) are endogenous ligands for LGR4 and LGR5. A fusion gene construct (mRspo1-Fc), encoding the mature form of mouse Rspol and the Fc fragment of mouse IgG2a, is biologically active (24). When incubating cells expressing LGR4 or LGR5 with mRspol- $\mathrm{Fc}$ at $4^{\circ} \mathrm{C}$ (to prevent internalization), a strong signal indicative of binding was observed on the cell surface. Wholecell competition binding assay showed that recombinant Rspo1-4 could compete with mRspol-Fc for binding to LGR4 and LGR5 (25). The results of binding analyses indicate that Rspo1-4 can bind to LGR4 and LGR5 with Rspo2 having the highest affinity to both receptors. Using $\beta$-catenin-responsive reporter assay (26), cells transfected with LGR4 or LGR5 displayed a dramatic increase in the potencies of Rspo1-4, ranging from 10- to 1,000-fold, on $\mathrm{Wnt} / \beta$-catenin signaling in the presence of exogenous Wnt3a (25).

Experiments using an unbiased screening strategy have also identified LGR4 and LGR5 as receptors of Rspo proteins (27). Depletion of LGR4 completely abolishes Rspol signaling, while overexpression of LGR4 potentiates Rspo1-4 signaling. Rspo1 interacts with the extracellular domain of LGR4 and LGR5 (27). Further, Rspol does not induce coupling between LGR4 and heterotrimeric G proteins, suggesting that LGR4 transmits Rspo signaling through a novel undefined mechanism independent of G protein signaling. This likely contributes to the relatively long time taken to deorphanize LGR4. This study further supports the conclusion that Rspo potentiates Wnt/ $\beta$-catenin signaling through LGR4 and LGR5, which is described in detail in Section "Intracellular Signaling of LGR4."

\section{Binding of R-Spondins to LGR4/5}

The extracelluar domain (ECD) of LGR4 exhibits a twisted horseshoe-like structure. Rspol adopts a flat and fold architecture and is bound in the concave surface of LGR4 through electrostatic and hydrophobic interactions (28). All the Rspo1-binding residues are conserved in LGR4-6, suggesting that LGR4-6 bind $\mathrm{R}$-spondins through an identical surface. R-spondin proteins have the same structural organization. They have two adjacent furinlike domains (FU1 and FU2) at the amino terminus, and a thrombospondin (TSP) domain close to the carboxyl terminus (29) (Figure 1). Sequence similarities of furin-like domains and TSP domain of four Rspo proteins from different species are high, for example, the identities of Rspol protein sequences between human and mouse is $94 \%$ (from Pubmed Blast), suggesting Rspondin proteins have conserved functions. A fragment containing two furin-like domains of R-spondin is sufficient to activate Wnt signaling, and both furin-like domains are required for the signaling activity of R-spondin $(18,30)$. In addition to LGR4/5, various membrane proteins have been reported to bind to Rspondin, including Wnt receptors Frizzled (20) and LRP6 (20, 

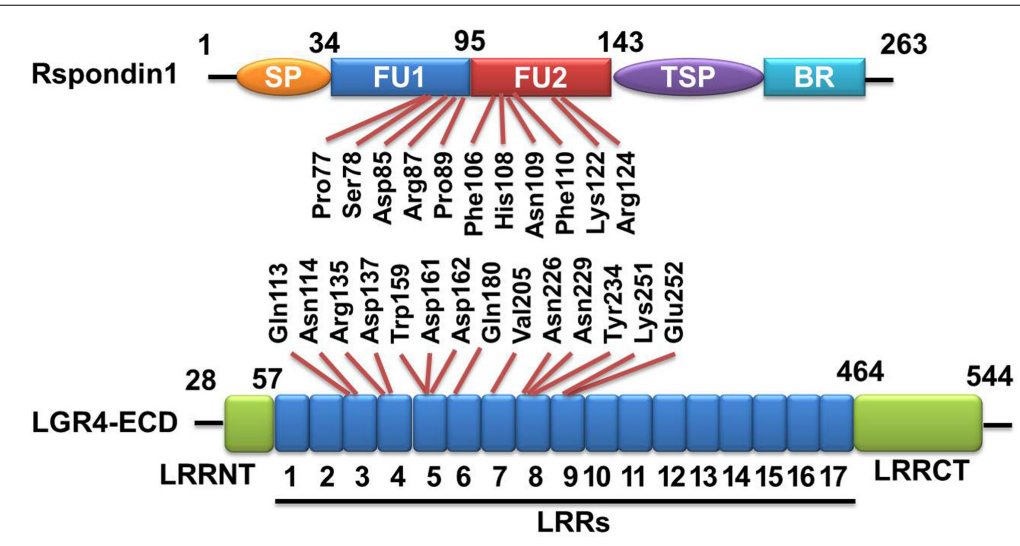

FIGURE 1 | Rspondin1-LGR4 linear sructure illustration. Linear structure of Rspondin1 and LGR4-ECD. Rspondin1 consists of a signal peptide (SP), two adjacent cysteine-rich furin-like domains (FU1/2), a common thrombospondin (TSP-1) motif and a basic amino acid-rich (BR) domain. LGR4 ectodomain
(LGR4-ECD) are characterized by 17 leucine-rich repeats (LRRs) flanked by the $\mathrm{N}$-terminal LRRNT region and the C-terminal LRRCT region. The main interacting residues of LGR4-ECD are LRR3-9 [refer to Ref. $(4,5)$ for detailed information].
31), Kremen (32), Syndecan 4 (33), and membrane E3 ubiquitin ligases ZNRF3/RNF43 (34). For example, Xie et al. reported that mutation of furin-like domain 1 (FU1) (R66A or Q71A) abolished the interaction between Rspol and ZNRF3, without affecting the interaction between Rspol and LGR4, while mutation of two residues of the furin-like domain 2 (FU2) (F106A or F110A) blocked the interaction between Rspo1 and LGR4, without affecting the interaction between Rspol and ZNRF3 (29). These results suggest that Rspo1 binds to ZNRF3 and LGR4 through distinct domains: the FU1 domain is involved in ZNRF3 binding, whereas the FU2 domain is involved in LGR4 binding. In the absence of Rspo, ZNRF3/RNF43 ubiquitinates the FZD receptors for degradation, resulting in low Wnt signaling activity. Rspol can bind to both ZNRF3 and LGR4 to induce their dimerization (34). In the Rspondin-LGR4-ZNRF3 complex, LGR4 serves as the engagement receptor to recruit R-spondin to ZNRF3. ZNRF3 serves as the effector receptor. Inhibition of ZNRF3 by R-spondin potentiates Wnt signaling. The R-spondin-LGR4/5-ZNRF3/RNF43 complex represents a fascinating example of a secreted protein regulating receptor turnover by targeting membrane E3 ubiquitin ligases (29) (Figure 2).

\section{Intracellular Signaling of LGR4}

Studies have suggested that interaction between Rspo proteins and LGR4 potentiates canonical Wnt/ $\beta$-catenin signaling, but does not activate Gi, Gs, or Gq pathways. Structurally, LGR4 and LGR5 are quite similar to other LRR-containing GPCRs which are coupled to heterotrimeric $G$ protein signaling by ligand binding. The intracellular signaling pathway by which Rspo and LGR4 potentiate $\mathrm{Wnt} / \beta$-catenin signaling remains largely unknown.

By examining proteins co-immunoprecipitated with LGR4, Kendra et al. identified IQGAP1 and IQGAP3 as potential candidates that could mediate the intracellular signaling of Rspo-LGR4 to the Wnt signalosome (35). Interaction between LGR4 and IQGAP1 occurs between the 7TM domain of LGR4 and the rasGAP-related domain (GRD) of IQGAP1. Stimulation of LGR4 by Rspos increases the affinity of IQGAP1 to

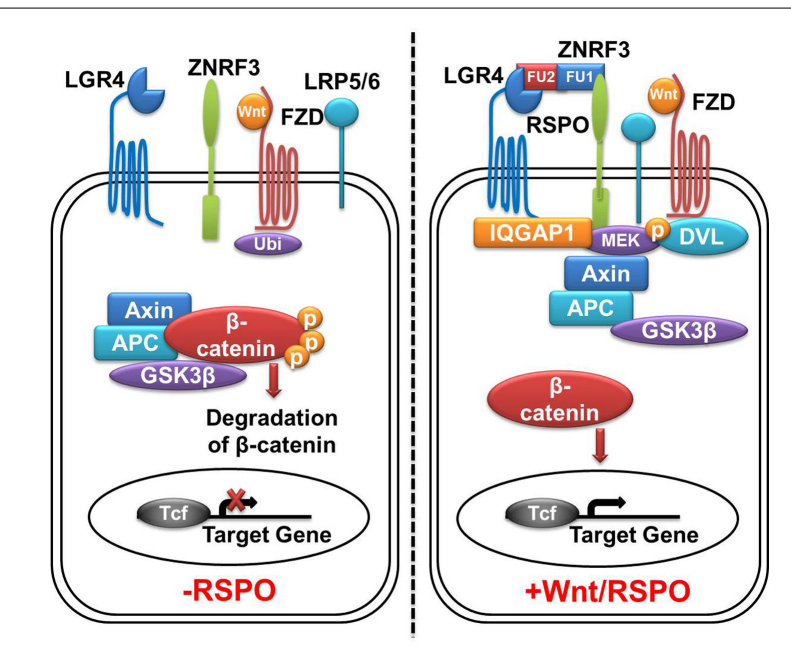

FIGURE 2 | LGR4-Wnt/ $\beta$-catenin signaling pathway. In the absence of Rspos, membrane E3 ubiquitin ligases ZNRF3/RNF43 ubiquitinates the (Frizzled) FZD receptor for degradation, Wnt signaling activity is blunted. Cytoplasmic $\beta$-catenin is degradated by the $\beta$-catenin destruction complex, leading to no $\beta$-catenin complex formation with T-cell transcription factor (Tcf) and subsequent silence in active transcriptional response. In the presence of Rspos, simultaneous binding of LGR4 and ZNRF3 inhibits the ubiquitination of FZD receptor, meanwhile, LGR4 recruits IQGAP1 and increases its affinity to DVL, leading to the formation of supercomplex with Wnt signalosome. This allows $\beta$-catenin accumulation in cytoplasm, followed by translocation into the nucleus and activation of TCF target genes. LGR4, leucine-rich repeat-containing G protein-coupled receptors 4; ZNRF3, zinc and ring finger 3; FZD, Frizzled class receptor; Rspos, R-spondins; LRP5/6, low-density lipoprotein receptor-related protein 5/6; Ubi, ubiquitination; DVL, disheveled.

disheveled (DVL), leading to the formation of a supercomplex between Rspo-LGR4 and the Wnt signalosome (35). Potentiation of Wnt signaling requires the MEK1/2-binding domain of IQGAP1, which provides the hint that LGR4-bound IQGAP1 brings in MEK1/2 to phosphorylate LRP6. In this configuration, IQGAP1 not only engages MEK1/2 to phosphorylate LRP5/6 but significantly enhance canonical Wnt signaling. IQGAP1 also recruits actin-polymerization complexes through binding to 
neural Wiskott-Aldrich syndrome protein (N-WASP) and mDial to coordinate actin dynamics (35), which is critical to the control of cell adhesion and migration (36-38). This dual-mechanism model provides an explanation for the pleiotropic functions of Rspo-LGR4 signaling in normal and cancer development, particularly for the crucial role of LGR4 in tubule elongation and branching in multiple organs (Figure 2).

Other signaling pathways have also been reported for LGR4. LGR4 (GPR48) participates in the development of the male epididymis and efferent ducts through regulation of ERalpha expression via the cAMP/PKA signaling pathway (39). LGR4 (GPR48) knockout suppressed ATF4, a key transcription factor in erythropoiesis, in midgestation fetal livers through the cAMP-PKA-CREB pathway, suggesting that GPR48 regulates erythropoiesis through ATF4 (40).

\section{LGR4 in Intestinal Development}

\section{Expression in Stem Cells}

A common feature of the LGR4/5/6 receptors is their expression in distinct types of adult stem cells. LGR5 is a marker for resident stem cells in Wnt-dependent compartments, including the small intestine, colon, stomach, and hair follicles (9). LGR6 serves as a marker of multipotent stem cells in the epidermis (12). LGR4 is widely expressed in proliferating cells (41).

The expression of LGR4 was investigated using an animal model with disrupted LGR4 gene by a trap vector carrying two biological markers, $\beta$-geo (a fusion between bacterial $\beta$ galactosidase and neomycin phosphotransferase) and a placental alkaline phosphatase (PLAP) (42). Due to the perinatal lethality of homozygosity for this insertion, LacZ and PLAP activity patterns in heterozygous mice were investigated. In embryonic day (E) 14 embryos, LGR4 was strongly labeled in eyes, tongue surface, olfactory epithelium and vomeronasal organ, ribs, esophagus, cartilage condensation of vertebrae, umbilical cord, medulla oblongata, pons, and Rathke's pouch (41). In adults, strong LacZ staining signals were detected in cartilage, kidney, adrenal gland, salivary glands, and testis, while lower intensity was observed in a wide range of organs (41).

\section{LGR4 in Digestive System}

At E15 and at birth, LacZ activity was detected in the pseudostratified epithelium and intervillus progenitors, respectively. In adults, epithelial expression of LGR4 was found along the crypts, but not in the villi by using X-gal staining and in situ hybridization (41). In the crypts, LGR4 expression was found above the Panethcell zone, in the transit-amplifying (TA) cell region, in crypt basal columnar cells, and co-localized with some Paneth cells. Outside the epithelium, LGR4 is expressed in the mesenchyme and smooth-muscle layers, intestinal subepithelial myofibroblasts and enteric neurons. A similar expression pattern was found in the duodenum and colon (41).

Another study has validated the presence of LGR4 immunoreactivity in Paneth cells which were distinguished by large secretory granules in the cytoplasm (43). Similar staining was also noted in crypt stem cells which are sandwiched between Paneth cells (44). No significant staining of LGR4 was found beyond the cells in the crypts. The intense LGR4 positive staining signal observed in Paneth cells and stem cells in the crypt region of mouse intestine were consistent with that of LGR4 mRNA distribution determined using lac- $Z$ alleles (45). In the mouse colon, only diffuse, weak LGR4 immunoreactivity in the cytoplasm was found in all cells from the base to the epithelial surface, with slightly stronger staining at the surface. No distinct staining was found in stem cells located at the crypt base in colon (43).

Intense LGR4 immunoreactivity was also found in the pancreatic islets with no staining in acinar cells. Co-staining of anti-LGR4 and anti-insulin antibody on mouse pancreas sections showed that LGR4 was expressed in all $\beta$-cells (43). Moreover, among the three LGR receptors, only LGR4 is expressed in the pancreas based on the analysis of EST data and Northern blotting (46). The intense staining of LGR4 in the islets strongly suggests that LGR4 mediates the effects of its endogenous ligands in the pancreas.

\section{LGR4 in Intestinal Development}

Intestinal crypts contain LGR $5^{+}$stem cells and their TA daughter cells, as well as terminally differentiated Paneth cells. Cells exiting crypts terminally differentiate into enterocytes, goblet cells, Mcells, Tuft cells, and enteroendocrine cells, and move up the flanks of the villi to undergo apoptosis upon reaching the villus tips (47). Paneth cells escape the crypt-villus flow by migrating to crypt bottoms where they persist for several weeks (48).

Mice homozygous for the gene trap LacZ knock-in allele, referred to as "Lgr4 knockout", displayed a hypomorphic phenotype with intrauterine growth retardation and perinatal lethality. Heterozygous mice with a LacZ gene trap knocked in the Lgr4 locus (42) have also been used. Although the timing of crypt development was normal in Lgr4-knockout mice, reduction in the crypt depth and epithelial cell proliferation were obvious from postnatal day (P) 15 (49). Differentiation of absorptive, enteroendocrine, and goblet-cell lineages was not modified significantly. However, defects in Paneth-cell differentiation were observed at all postnatal stages, with reduction in Paneth-cell number at P21 and decreased expression of the terminal differentiation markers P-lyzozyme and cryptdin 4 (49), suggesting a key role for LGR4 in postnatal epithelial cell proliferation and terminal Paneth-cell differentiation.

Ex vivo experiments have demonstrated that LGR4 is required for the maintenance of crypt stem cells. Crypts cultured from P15 wild-type or heterozygous mice differentiated into multifingered organoids after 3 days in culture. After generation of hollow spheres containing mainly stem and TA cells (day 0.5-1), structures grown from P15 Lgr4-knockout intestine did not increase further in size and became filled with cellular material (days 1.5-2) (49). Lgr4-knockout organoids started to disaggregate between days 2 and 3, and died before day 7 . The same phenotype was observed in Lgr4-knockout progenitors isolated from newborn mice, when fully differentiated Paneth cells are not yet present (49).

\section{LGR4 in Energy Metabolism}

The presence of LGR4 in hypothalamic energy homeostatic areas and its co-localization with key energy homeostatic neurons suggests that it may contribute to the regulation of energy 
homeostasis. In situ hybridization revealed that LGR4 mRNA is highly expressed in the cortex, hippocampus, amygdala, and the hypothalamus (41). In the cortex, LGR4 mRNA is expressed in layers II and III. In the hippocampus, LGR4 is expressed in CA1, CA2, CA3, and the dentate gyrus (DG).The habenular nuclei (Hbs) of the epithalamus also express LGR4. In the amygdala, LGR4 mRNA is expressed with high levels in the medial amygdaloid nucleus, posteroventral nucleus, and basal lateral amygdaloid nucleus (41). In the hypothalamus, LGR4 is expressed in the ventromedial hypothalamus (VMH), the arcuate nucleus (ARC), median eminence (ME), and the ependymocytes lining the third ventricle (50). The ME and ependymocytes have the highest levels of LGR4 expression, followed by $\mathrm{VMH}$ and ARC. The expression pattern of LGR4 in the VMH overlaps that of brain-derived neurotrophic factor (BDNF) (51). Double in situ hybridization showed that in the ARC, LGR4 is expressed by most neuropeptide Y (NPY) neurons and proopiomelanocortin (POMC) neurons. NPY neurons express higher levels of LGR4 compared with POMC neurons in the ARC. All POMC neurons in the ME express LGR4 at the highest levels. In the VMH, LGR4 is expressed by the majority of BDNF neurons (50).

Rspo1 and Rspo3, ligands of LGR4, are expressed in hypothalamic energy homeostatic areas (50). Their levels were downregulated by fasting and up-regulated by the satiety factor insulin, indicating that they might be involved in the regulation of energy homeostasis as anorexigenic factors. The inhibition of food intake observed after intracerebroventricular injection of Rspol or Rspo3 support this concept (50). Rspo1 is more potent than Rspo3 in inhibiting food intake. Consistent with this observation, Rspo1 binds to LGR4 with an affinity higher than Rspo3 (25).

GPR48 (LGR4) is critical in development, and Gpr48 mutant mice display early neonatal lethality. Wang et al. have established the Gpr48 (LGR4) hypomorphic mutant mice by microinjecting gene trap-mutated Gpr48 ES cells into C57BL/6 blastocysts. The insertion of the trap vector into intron 1 of the Gpr48 gene resulted in approximately $90 \%$ knockdown efficiency in the kidney and adrenal gland of adult LGR4 mutant mice. Approximate half of LGR4 mutant newborns died within $28 \mathrm{~h}$ after birth, but no further deaths occurred in the following $20 \mathrm{~h}$ (52). Studies of the Lgr4-knockout mice have revealed a critical role of LGR4 in lipid metabolism. Relative to wild-type mice fed normal chow diet, both male and female Lgr4 mutant mice exhibited decreased body weight and body fat content, including epididymal white adipose tissue (eWAT) and inguinal WAT (iWAT), whereas brown adipose tissue (BAT) content remained unaltered (53). Consistent with the lean phenotype, Lgr4 mutant mice showed improved glucose tolerance and reduced fasting total cholesterol levels. When challenged with a high-fat diet (HFD), both male and female Lgr4 mutant mice showed a resistance to HFD-induced body weight gain, with improved glucose tolerance and insulin sensitivity (53). Higher $\mathrm{O}_{2}$ consumption, $\mathrm{CO}_{2}$ production, and body temperature indicated that energy expenditure was elevated in Lgr4 mutant mice. Compared to the white, large, and unilocular adipocytes comprising WAT in wild-type mice, Lgr4 mutant mice showed reduced WAT mass with beige color, smaller, and multilocular adipocytes containing increased mitochondrion number. These results indicate an adipocyte phenotype transformation in Lgr4 mutant mice (52). Consistent with this concept, Ucp1 and other thermogenic genes, including Pgc-1 $\alpha$, Cidea, cytochrome c, Cpt2, and Nrf1, were significantly increased in eWAT of Lgr4 mutant mice, and were further enhanced under cold stress or isoprenaline treatment. Beige cell markers CD137 and TMEM26 (54) were also increased in eWAT of Lgr4 mutant mice after isoprenaline treatment. These results demonstrate that $\operatorname{Lr} 4$ ablation drives the acquisition of functional brown-like adipocytes in the WAT depots, leading to increased energy expenditure (52).

The association of LGR4 with human obesity has been demonstrated by a case-control study of early-onset obesity in which four SNPs, located in the encoding and flanking regions of the LGR4 locus, were found to be significantly correlated with body mass index. A low-frequency non-synonymous LGR4 variant (A750T) was identified more than twice as commonly in obese patients when compared with controls (52). This site in LGRs is highly conserved among different species, and constitutively activated point mutations of the corresponding site in LHR, TSHR, and FSHR have been reported (55-57). The A750T variant showed higher stimulating activity of a CRE-luciferase reporter than wild-type LGR4, suggesting a functional variation. All these observations suggest a contribution of LGR4 to human adiposity.

Another study based on the LGR4 mutant mice described before also indicates a correlation between LGR4 and lipid metabolism (58) in a circadian rhythm-related manner. Resting energy expenditure (RER) is higher in the dark phase than in the light phase in wild type (WT) mice (59), suggesting the existence of a circadian rhythm in substrate utilization for energy during the day, more glucose in the dark phase, and more lipid usage in the light phase. In Lgr4 mutant mice, the RER was higher than that of their WT littermates during the dark phase with no difference during the light phase (57), suggesting that lack of LGR4 altered the circadian rhythm of lipid metabolism. Lgr4-knockout mice consumed less lipids but more sugar compared with WT mice (60, 61). Lgr4 mutant mice exhibited higher plasma triglyceride levels and lost the rhythmic pattern compared with WT mice. Lgr4 mutant mice also presented a change in plasma non-esterified fatty acid levels, reflected by lower plasma levels during the light phase and higher levels in the dark phase in comparison with WT mice. Interestingly, loss of LGR4 does not affect clock gene expression in the liver. In WT mice, LGR4 expression in liver was higher during the light phase than the dark phase, presenting a peak at ZT4 and a nadir at ZT16, indicating a circadian rhythm. Lgr4 expression levels in Lgr4 mutant mice were very low and amplitude was dampened (57). Lack of LGR4 causes an arrhythmic plasma lipid phenotype in mice.

\section{Therapeutics Potential of Rspos-LGR4}

\section{Treatment for Inflammatory Bowel Disease}

As defective epithelial restitution is an important risk factor for inflammatory bowel disease (IBD), it is not surprising that dysfunction of genes involved in intestinal development, proliferation, and differentiation will increase susceptibility to IBD. 
When Lgr4 hypomorphic mice are subjected to the dextran sulfate sodium (DSS)-induced IBD, a more severe colitis developed in Lgr4 mutant mice than in WT sex-matched littermates. Higher body weight loss, a hallmark of intestinal inflammation, was observed in Lgr4 mutant mice. This observation was concordant with a more severe anemia (45). Although the small intestine is not the major target of DSS-induced tissue damage (62), the relative length reduction was significantly increased in Lgr4 mutant mice, indicating critical functions of Lgr4 in the small intestine. Almost all crypts throughout the small intestine were lost in Lgr4 mutant mice but remained intact in WT littermates. Histological examination showed dramatically increased signs of colitis, which is characterized by the loss of crypts and the infiltration of leukocytes into the colons of Lgr4 mutant mice (45). Infiltration of neutrophils was significantly higher in the colon of Lgr4 mutant mice. Additionally, inflammatory cytokines, such as TNF $\alpha$, IL6, and IL1, were significantly increased in Lgr4 mutant mice after DSS administration, suggesting a more severe inflammatory response (45). Significant decreases in Ki67positive proliferating cells were observed in Lgr4 mutant intestines during tissue regeneration. No significant alteration of apoptosis was observed either in control conditions or in the recovery period, indicating that LGR4 is responsible for epithelial cell proliferation but not apoptosis during DSS-induced tissue regeneration (45).

Human Rspo1 protein effectively increases survival and proliferation of LGR $5^{+}$intestinal stem cells in vitro through activation of Wnt/ $\beta$-catenin signaling (63). The mitogenic activity of Rspo1 on intestinal stem cells may be useful in the therapy of IBD because of its stimulating effect on crypt cell growth to accelerate mucosal regeneration. In both acute and chronic phases of colitis in mouse models, administration of Rspol protein preserves mucosal integrity in both small and large bowel by stimulating crypt epithelial cell mitosis (64).

\section{Protective Effects of R-Spondin in Chemoradiotherapy-Induced Gut Injury}

Stimulation of Wnt/ $\beta$-catenin signaling with Rspol can ameliorate 5-fluorouracil (5-FU) and radiation-induced gut damage, including radiation-induced gastrointestinal syndrome (RIGS) (64-66). Recently, Zhou et al. have found that combination of Slit2 and Rspol could potentially protect gut from chemoradiotherapyinduced damage (67). Slit is a secreted protein which functions through the TM protein Roundabout (Robo) receptor as a chemorepellent in axon guidance and neuronal migration, and as an inhibitor in leukocyte chemotaxis (68). The therapeutic dosage of 5-FU (19), a well-characterized chemotherapy agent, markedly shortened villus length, reduced numbers of LGR5 $^{+}$intestinal stem cells, and $\mathrm{Ki}^{+}{ }^{+}$transient amplifying cells in jejunum. Using Lgr5-enhanced green fluorescent protein (eGFP)-internal ribosome entry site (IRES)-CreERT2 (Lgr5-GFP) mice to detect the intestine stem cells (10), the lethal dose of 5-FU has been found to abolish $>90 \%$ of GFP high $^{\text {his }}$ intestinal stem cells. However, a 3-day treatment of rSlit2 or rRspol alone protected $40 \%$ of $\mathrm{GFP}^{\text {high }}$ stem cells. Combination

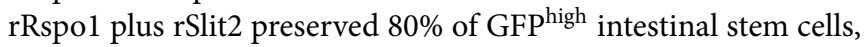
indicating that Slit2 acts synergistically with Rspo, leading to prolongation of overall survival after exposure to lethal doses of chemotherapy (67).

$\mathrm{Apc}^{\mathrm{Min} /+}$ mice with spontaneous intestinal adenomas were treated with DSS to induce inflammation-related intestinal carcinogenesis, a murine model of multifactorial human colorectal cancer (CRC) (69). Administration of DSS-treated Apc ${ }^{\mathrm{Min} /+}$ mice with rSlit2 or rRspo1 alone led to a $20-30 \%$ survival rate upon the lethal dosage of 5-FU. Combination of rSlit2 and rRspol led to a $60 \%$ survival rate (67), demonstrating functional cooperation between Slit2 and Rspo1. Concomitant prolongations of the villus length, augmentations of $\operatorname{Lgr} 5^{+}$stem cells and $\mathrm{Ki}^{+} 7^{+}$transient amplifying cells in the jejunum were observed when animals were treated with a combination of rSlit2 plus rRspo1 (67).

\section{R-Spondin Fusion Protein in the Treatment of Colorectal Cancer}

Colorectal cancer is the fourth most prevalent cancer, accounting for over 50,000 deaths per year in the United States. Approximately $15 \%$ of CRCs have microsatellite instability arising from defects in the DNA mismatch-repair system, whereas the other $85 \%$ of microsatellite-stable CRCs are the result of chromosomal instability (70).

Using RNA-seq data, Seshagiri et al. have identified 36 rearrangements that result in gene fusions (71), including two recurrent ones. The recurrent fusions found in microsatellitestable samples involve the R-spondin family members, Rspo 2 and Rspo3 (70). Both of them were expected to produce functional Rspos protein. The expression of Rspo 2 and Rspo 3 in colon tumor samples, containing the fusions, was elevated compared with the tumor samples lacking R-spondin fusions (70). Furthermore, all of the Rspo-positive fusion tumors expressed the potential R-spondin receptors LGR4, LGR5, and LGR6. Additionally, alteration of the Rspo2 gene is linked to CRC in a transposonbased genetic screening in mice (72).Consistent with the elevated expression of Rspo genes observed in human tumors, a 20-fold increase in Rspo2 messenger RNA expression in a mouse tumor carrying a transposon insertion near the Rspo2 transcription start site was detected relative to adjacent normal tissue. These observations indicate that the R-spondins may function as drivers in human CRCs (70). Although further studies are required to fully understand the role of R-spondin fusions in CRC development, they represent attractive targets for antibody-based therapy in CRC patients positive for R-spondin fusions. Other therapeutic strategies that target downstream components of the Wnt signaling cascade might be effective against tumors positive for R-spondin fusions.

\section{R-Spondin in the Treatment of Diabetes}

The development of type 2 diabetes mellitus (T2DM) usually requires the presence of insulin resistance, impaired $\beta$-cell function, and the loss of $\beta$-cells (73). Type 1 diabetes mellitus (T1DM) is characterized by autoimmune-mediated destruction of $\beta$-cells. Novel therapeutic approaches might include expanding $\beta$-cell mass. As reported by Wong et al., Rspol enhances insulin mRNA levels after stimulation for $12 \mathrm{~h}$. Rspol can also regulate insulin secretion in mouse islets. Static incubation of islets with Rspol for $2 \mathrm{~h}$ induced a significant increase in insulin secretion in a 
TABLE 1 | Roles of LGR4 in intestinal functions and energy metabolism, and summary of the therapeutic potentials of Rspos-LGR4 system.

\begin{tabular}{|c|c|c|}
\hline $\begin{array}{l}\text { Roles of } \\
\text { LGR4 }\end{array}$ & Physiological functions & Therapeutics potentials \\
\hline In intestine & $\begin{array}{l}\text { 1. Postnatal epithelial cell } \\
\text { proliferation and terminal } \\
\text { Paneth-cell differentiation } \\
\text { 2. Maintenance of crypt } \\
\text { stem cells }\end{array}$ & $\begin{array}{l}\text { 1. Inflammatory bowel disease } \\
\text { 2. Chemoradiotherapy-induced } \\
\text { gut injury } \\
\text { 3. Colorectal cancer }\end{array}$ \\
\hline $\begin{array}{l}\text { In energy } \\
\text { metabolism }\end{array}$ & $\begin{array}{l}\text { 1. Inhibition of food intake } \\
\text { 2. Acquisition of functional } \\
\text { brown-like adipocytes in the } \\
\text { WAT depots in } \mathrm{Lgr}^{-/-} \text {mice } \\
\text { 3. Association with human } \\
\text { obesity } \\
\text { 4. Arrhythmic plasma lipid } \\
\text { phenotype in } \mathrm{Lgr}^{-/-} \text {mice }\end{array}$ & $\begin{array}{l}\text { 1. Obesity } \\
\text { 2. Diabetes mellitus } \\
\text { 3. Lipid metabolism }\end{array}$ \\
\hline
\end{tabular}

glucose-independent manner (74). Treatment with recombinant mouse Rspo1 also increases MIN6-cell proliferation. Rspo1 induced an increase in BrdU incorporation in insulin-positive cells (74). When treated MIN6 cells were exposed to a mixture of cytokines for $18 \mathrm{~h}$, the level of activated, cleaved caspase 3 was significantly increased. The increase in cleaved caspase 3 was prevented by pretreatment with Wnt3a, as well as by Rspo1. A similar observation was made in dispersed murine $\beta$-cells. Treatment with cytokines for $18 \mathrm{~h}$ significantly increased the number of TUNEL-positive $\beta$-cells; pretreatment with Rspol significantly reduced cytokine-induced apoptosis (74). These observations suggest that Rspol may be a potential novel molecule for the treatment of patients with T2DM or T1DM.

\section{References}

1. Luo CW, Hsueh AJ. Genomic analyses of the evolution of LGR genes. Chang Gung Med J (2006) 29:2-8.

2. Kobe B, Deisenhofer J. Crystal structure of porcine ribonuclease inhibitor, a protein with leucine-rich repeats. Nature (1993) 366:751-6. doi:10.1038/ 366751a0

3. Kajava AV. Structural diversity of leucine-rich repeat proteins. J Mol Biol (1998) 277:519-27. doi:10.1006/jmbi.1998.1643

4. Wang D, Huang B, Zhang S, Yu X, Wu W, Wang X. Structural basis for Rspondin recognition by LGR4/5/6 receptors. Genes Dev (2013) 27:1339-44. doi:10.1101/gad.219360.113

5. Xu K, Xu Y, Rajashankar KR, Robev D, Nikolov DB. Crystal structures of Lgr4 and its complex with R-spondin1. Structure (2013) 21:1683-9. doi:10.1016/j.str. 2013.07.001

6. Barker N, Tan S, Clevers H. Lgr proteins in epithelial stem cell biology. Development (2013) 140:2484-94. doi:10.1242/dev.083113

7. Van Loy T, Vandersmissen HP, Van Hiel MB, Poels J, Verlinden H, Badisco $\mathrm{L}$, et al. Comparative genomics of leucine-rich repeats containing $\mathrm{G}$ proteincoupled receptors and their ligands. Gen Comp Endocrinol (2008) 155:14-21. doi:10.1016/j.ygcen.2007.06.022

8. Hsu SY, Kudo M, Chen T, Nakabayashi K, Bhalla A, van der Spek PJ, et al. The three subfamilies of leucine-rich repeat-containing $G$ proteincoupled receptors (LGR): identification of LGR6 and LGR7 and the signaling mechanism for LGR7. Mol Endocrinol (2000) 14:1257-71. doi:10.1210/mend. 14.8.0510

9. Barker N, Clevers H. Leucine-rich repeat-containing G-protein-coupled receptors as markers of adult stem cells. Gastroenterology (2010) 138:1681-96. doi: 10.1053/j.gastro.2010.03.002

10. Barker N, van Es JH, Kuipers J, Kujala P, van den Born M, Cozijnsen M, et al. Identification of stem cells in small intestine and colon by marker gene Lgr5. Nature (2007) 449:1003-7. doi:10.1038/nature06196

\section{Conclusion}

The importance of LGR4 and its ligands-Rspos in the regulation of canonical and non-canonical Wnt signaling pathways has been established in a variety of in vitro and in vivo studies using animal models and human genetic analysis. Although significant progress has been made in our understanding of how Rspo binds with LGR4 and regulates the Wnt signaling pathway at the molecular level, the following fundamental questions remain unanswered. How does LGR4 interact with FZD (Wnt receptor) after binding with Rspo proteins? Does LGR4 have a specific intracellular signaling pathway or simply function as a potentiator of Wnt signaling? Additionally, discrepancy between gain and loss of LGR4 function exists. While LGR4-knockout mice showed an improvement in glucose metabolism, Rspol has been reported to significantly induce $\beta$-cell proliferation and insulin secretion. Further studies on the functions and signaling mechanisms of the LGR4 and Rspo proteins will facilitate the development of therapeutic strategy for human diseases, such as IBD, CRC, and diabetes, by targeting Rspo-LGR4 (Table 1).

\section{Author Contributions}

ZL, WZ, and MM wrote, discussed, and edited the manuscript.

\section{Acknowledgments}

This work was supported by grants from the National Natural Science Foundation of China $(81330010,81390354)$ and American Diabetes Association grant \#1-13-BS-225.

11. Jaks V, Barker N, Kasper M, van Es JH, Snippert HJ, Clevers H, et al. Lgr5 marks cycling, yet long-lived, hair follicle stem cells. Nat Genet (2008) 40:1291-9. doi:10.1038/ng.239

12. Snippert HJ, Haegebarth A, Kasper M, Jaks V, van Es JH, Barker N, et al. Lgr6 marks stem cells in the hair follicle that generate all cell lineages of the skin. Science (2010) 327:1385-9. doi:10.1126/science.1184733

13. Clevers H, Nusse R. Wnt/beta-catenin signaling and disease. Cell (2012) 149:1192-205. doi:10.1016/j.cell.2012.05.012

14. Niehrs C. The complex world of WNT receptor signalling. Nat Rev Mol Cell Biol (2012) 13:767-79. doi:10.1038/nrm3470

15. Hauser F, Sondergaard L, Grimmelikhuijzen CJ. Molecular cloning, genomic organization and developmental regulation of a novel receptor from Drosophila melanogaster structurally related to gonadotropin-releasing hormone receptors for vertebrates. Biochem Biophys Res Commun (1998) 249:822-8. doi:10.1006/ bbrc. 1998.9230

16. Nothacker HP, Grimmelikhuijzen CJ. Molecular cloning of a novel, putative G protein-coupled receptor from sea anemones structurally related to members of the FSH, TSH, LH/CG receptor family from mammals. Biochem Biophys Res Commun (1993) 197:1062-9. doi:10.1006/bbrc.1993.2586

17. McDonald T, Wang R, Bailey W, Xie G, Chen F, Caskey CT, et al. Identification and cloning of an orphan $\mathrm{G}$ protein-coupled receptor of the glycoprotein hormone receptor subfamily. Biochem Biophys Res Commun (1998) 247:266-70. doi:10.1006/bbrc.1998.8774

18. Kazanskaya O, Glinka A, del Barco Barrantes I, Stannek P, Niehrs C, Wu W. Rspondin2 is a secreted activator of Wnt/beta-catenin signaling and is required for Xenopus myogenesis. Dev Cell (2004) 7:525-34. doi:10.1016/j.devcel.2004. 07.019

19. Kim KA, Kakitani M, Zhao J, Oshima T, Tang T, Binnerts M, et al. Mitogenic influence of human R-spondin1 on the intestinal epithelium. Science (2005) 309:1256-9. doi:10.1126/science.1112521

20. Nam JS, Turcotte TJ, Smith PF, Choi S, Yoon JK. Mouse cristin/R-spondin family proteins are novel ligands for the frizzled 8 and LRP6 receptors and activate 
beta-catenin-dependent gene expression. J Biol Chem (2006) 281:13247-57. doi:10.1074/jbc.M508324200

21. Kim KA, Wagle M, Tran K, Zhan X, Dixon MA, Liu S, et al. R-spondin family members regulate the Wnt pathway by a common mechanism. Mol Biol Cell (2008) 19:2588-96. doi:10.1091/mbc.E08-02-0187

22. Kim KA, Zhao J, Andarmani S, Kakitani M, Oshima T, Binnerts ME, et al. R-spondin proteins: a novel link to beta-catenin activation. Cell Cycle (2006) 5:23-6. doi:10.4161/cc.5.1.2305

23. de Lau WB, Snel B, Clevers HC. The R-spondin protein family. Genome Biol (2012) 13:242. doi:10.1186/gb-2012-13-3-242

24. Ootani A, Li X, Sangiorgi E, Ho QT, Ueno H, Toda S, et al. Sustained in vitro intestinal epithelial culture within a Wnt-dependent stem cell niche. Nat Med (2009) 15:701-6. doi:10.1038/nm.1951

25. Carmon KS, Gong X, Lin Q, Thomas A, Liu Q. R-spondins function as ligands of the orphan receptors LGR4 and LGR5 to regulate Wnt/beta-catenin signaling. Proc Natl Acad Sci U S A (2011) 108:11452-7. doi:10.1073/pnas. 1106083108

26. Veeman MT, Slusarski DC, Kaykas A, Louie SH, Moon RT. Zebrafish prickle, a modulator of noncanonical Wnt/Fz signaling, regulates gastrulation movements. Curr Biol (2003) 13:680-5. doi:10.1016/S0960-9822(03)00240-9

27. Ruffner H, Sprunger J, Charlat O, Leighton-Davies J, Grosshans B, Salathe A, et al. R-spondin potentiates Wnt/beta-catenin signaling through orphan receptors LGR4 and LGR5. PLoS One (2012) 7:e40976. doi:10.1371/journal. pone. 0040976

28. Xu JG, Huang C, Yang Z, Jin M, Fu P, Zhang N, et al. Crystal structure of LGR4Rspol complex: insights into the divergent mechanisms of ligand recognition by leucine-rich repeat G-protein-coupled receptors (LGRs). J Biol Chem (2015) 290:2455-65. doi:10.1074/jbc.M114.599134

29. Xie Y, Zamponi R, Charlat O, Ramones M, Swalley S, Jiang X, et al. Interaction with both ZNRF3 and LGR4 is required for the signalling activity of R-spondin. EMBO Rep (2013) 14:1120-6. doi:10.1038/embor.2013.167

30. Glinka A, Dolde C, Kirsch N, Huang YL, Kazanskaya O, Ingelfinger D, et al. LGR4 and LGR5 are R-spondin receptors mediating Wnt/beta-catenin and Wnt/PCP signalling. EMBO Rep (2011) 12:1055-61. doi:10.1038/embor. 2011.175

31. Wei Q, Yokota C, Semenov MV, Doble B, Woodgett J, He X. R-spondin1 is a high affinity ligand for LRP6 and induces LRP6 phosphorylation and beta-catenin signaling. J Biol Chem (2007) 282:15903-11. doi:10.1074/jbc.M701927200

32. Binnerts ME, Kim KA, Bright JM, Patel SM, Tran K, Zhou M, et al. R-spondin1 regulates Wnt signaling by inhibiting internalization of LRP6. Proc Natl Acad Sci U S A (2007) 104:14700-5. doi:10.1073/pnas.0702305104

33. Ohkawara B, Glinka A, Niehrs C. Rspo3 binds syndecan 4 and induces Wnt/PCP signaling via clathrin-mediated endocytosis to promote morphogenesis. Dev Cell (2011) 20:303-14. doi:10.1016/j.devcel.2011.01.006

34. Hao HX, Xie Y, Zhang Y, Charlat O, Oster E, Avello M, et al. ZNRF3 promotes Wnt receptor turnover in an R-spondin-sensitive manner. Nature (2012) 485:195-200. doi:10.1038/nature11019

35. Carmon KS, Gong X, Yi J, Thomas A, Liu Q. RSPO-LGR4 functions via IQGAP1 to potentiate Wnt signaling. Proc Natl Acad Sci U S A (2014) 111:E1221-9. doi:10.1073/pnas.1323106111

36. Brandt DT, Grosse R. Get to grips: steering local actin dynamics with IQGAPs. EMBO Rep (2007) 8:1019-23. doi:10.1038/sj.embor.7401089

37. Parsons JT, Horwitz AR, Schwartz MA. Cell adhesion: integrating cytoskeletal dynamics and cellular tension. Nat Rev Mol Cell Biol (2010) 11:633-43. doi:10. 1038/nrm2957

38. Bensenor LB, Kan HM, Wang N, Wallrabe H, Davidson LA, Cai Y, et al. IQGAP1 regulates cell motility by linking growth factor signaling to actin assembly. J Cell Sci (2007) 120:658-69. doi:10.1242/jcs.03376

39. Li XY, Lu Y, Sun HY, Wang JQ, Yang J, Zhang HJ, et al. G protein-coupled receptor 48 upregulates estrogen receptor alpha expression via cAMP/PKA signaling in the male reproductive tract. Development (2010) 137:151-7. doi: 10.1242/dev.040659

40. Song H, Luo J, Luo W, Weng J, Wang Z, Li B, et al. Inactivation of Gprotein-coupled receptor 48 (Gpr48/Lgr4) impairs definitive erythropoiesis at midgestation through down-regulation of the ATF4 signaling pathway. J Biol Chem (2008) 283:36687-97. doi:10.1074/jbc.M800721200

41. Van Schoore G, Mendive F, Pochet R, Vassart G. Expression pattern of the orphan receptor LGR4/GPR48 gene in the mouse. Histochem Cell Biol (2005) 124:35-50. doi:10.1007/s00418-005-0002-3
42. Leighton PA, Mitchell KJ, Goodrich LV, Lu X, Pinson K, Scherz P, et al. Defining brain wiring patterns and mechanisms through gene trapping in mice. Nature (2001) 410:174-9. doi:10.1038/35065539

43. Yi J, Xiong W, Gong X, Bellister S, Ellis LM, Liu Q. Analysis of LGR4 receptor distribution in human and mouse tissues. PLoS One (2013) 8:e78144. doi:10. 1371/journal.pone.0078144

44. Sato T, van Es JH, Snippert HJ, Stange DE, Vries RG, van den Born M, et al. Paneth cells constitute the niche for Lgr5 stem cells in intestinal crypts. Nature (2011) 469:415-8. doi:10.1038/nature09637

45. Liu S, Qian Y, Li L, Wei G, Guan Y, Pan H, et al. Lgr4 gene deficiency increases susceptibility and severity of dextran sodium sulfate-induced inflammatory bowel disease in mice. J Biol Chem (2013) 288:8794-803. doi:10.1074/jbc.M112. 436204

46. Hsu SY, Liang SG, Hsueh AJ. Characterization of two LGR genes homologous to gonadotropin and thyrotropin receptors with extracellular leucine-rich repeats and a G protein-coupled, seven-transmembrane region. Mol Endocrinol (1998) 12:1830-45. doi:10.1210/mend.12.12.0211

47. Clevers H. The intestinal crypt, a prototype stem cell compartment. Cell (2013) 154:274-84. doi:10.1016/j.cell.2013.07.004

48. Bierknes $\mathrm{M}$, Cheng $\mathrm{H}$. The stem-cell zone of the small intestinal epithelium. I. Evidence from Paneth cells in the adult mouse. Am J Anat (1981) 160:51-63. doi:10.1002/aja.1001600106

49. Mustata RC, Van Loy T, Lefort A, Libert F, Strollo S, Vassart G, et al. Lgr4 is required for Paneth cell differentiation and maintenance of intestinal stem cells ex vivo. EMBO Rep (2011) 12:558-64. doi:10.1038/embor.2011.52

50. Li JY, Chai B, Zhang W, Fritze DM, Zhang C, Mulholland MW. LGR4 and its ligands, R-spondin 1 and R-spondin 3, regulate food intake in the hypothalamus of male rats. Endocrinology (2014) 155:429-40. doi:10.1210/en.2013-1550

51. Larsen MH, Hay-Schmidt A, Ronn LC, Mikkelsen JD. Temporal expression of brain-derived neurotrophic factor (BDNF) mRNA in the rat hippocampus after treatment with selective and mixed monoaminergic antidepressants. Eur J Pharmacol (2008) 578:114-22. doi:10.1016/j.ejphar.2007.08.050

52. Wang J, Li X, Ke Y, Lu Y, Wang F, Fan N, et al. GPR48 increases mineralocorticoid receptor gene expression. J Am Soc Nephrol (2012) 23:281-93. doi:10.1681/ASN.2011040351

53. Wang J, Liu R, Wang F, Hong J, Li X, Chen M, et al. Ablation of LGR4 promotes energy expenditure by driving white-to-brown fat switch. Nat Cell Biol (2013) 15:1455-63. doi:10.1038/ncb2867

54. Wu J, Bostrom P, Sparks LM, Ye L, Choi JH, Giang AH, et al. Beige adipocytes are a distinct type of thermogenic fat cell in mouse and human. Cell (2012) 150:366-76. doi:10.1016/j.cell.2012.05.016

55. Yano K, Saji M, Hidaka A, Moriya N, Okuno A, Kohn LD, et al. A new constitutively activating point mutation in the luteinizing hormone/choriogonadotropin receptor gene in cases of male-limited precocious puberty. J Clin Endocrinol Metab (1995) 80:1162-8. doi:10.1210/jc.80.4.1162

56. Gozu HI, Bircan R, Krohn K, Muller S, Vural S, Gezen C, et al. Similar prevalence of somatic TSH receptor and Gsalpha mutations in toxic thyroid nodules in geographical regions with different iodine supply in Turkey. Eur $J$ Endocrinol (2006) 155:535-45. doi:10.1530/eje.1.02253

57. Achrekar SK, Modi DN, Meherji PK, Patel ZM, Mahale SD. Follicle stimulating hormone receptor gene variants in women with primary and secondary amenorrhea. J Assist Reprod Genet (2010) 27:317-26. doi:10.1007/s10815-0109404-9

58. Wang F, Zhang X, Wang J, Chen M, Fan N, Ma Q, et al. LGR4 acts as a link between the peripheral circadian clock and lipid metabolism in liver. $J \mathrm{Mol}$ Endocrinol (2014) 52:133-43. doi:10.1530/JME-13-0042

59. Tu Y, Thupari JN, Kim EK, Pinn ML, Moran TH, Ronnett GV, et al. C75 alters central and peripheral gene expression to reduce food intake and increase energy expenditure. Endocrinology (2005) 146:486-93. doi:10.1210/en.20040976

60. Hems DA, Rath EA, Verrinder TR. Fatty acid synthesis in liver and adipose tissue of normal and genetically obese (ob/ob) mice during the 24-hour cycle. Biochem J (1975) 150:167-73. doi:10.1042/bj1500167

61. Roberts R, Bickerton AS, Fielding BA, Blaak EE, Wagenmakers AJ, Chong MF, et al. Reduced oxidation of dietary fat after a short term high-carbohydrate diet. Am J Clin Nutr (2008) 87:824-31.

62. Elson CO, Sartor RB, Tennyson GS, Riddell RH. Experimental models of inflammatory bowel disease. Gastroenterology (1995) 109:1344-67. doi:10. 1016/0016-5085(95)90599-5 
63. Sato T, Vries RG, Snippert HJ, van de Wetering M, Barker N, Stange DE, et al. Single Lgr5 stem cells build crypt-villus structures in vitro without a mesenchymal niche. Nature (2009) 459:262-5. doi:10.1038/nature07935

64. Zhao J, de Vera J, Narushima S, Beck EX, Palencia S, Shinkawa P, et al. Rspondin1, a novel intestinotrophic mitogen, ameliorates experimental colitis in mice. Gastroenterology (2007) 132:1331-43. doi:10.1053/j.gastro.2007.02.001

65. Zhao J, Kim KA, De Vera J, Palencia S, Wagle M, Abo A. R-spondin1 protects mice from chemotherapy or radiation-induced oral mucositis through the canonical Wnt/beta-catenin pathway. Proc Natl Acad Sci U S A (2009) 106:2331-6. doi:10.1073/pnas.0805159106

66. Bhanja P, Saha S, Kabarriti R, Liu L, Roy-Chowdhury N, Roy-Chowdhury J, et al. Protective role of R-spondin1, an intestinal stem cell growth factor, against radiation-induced gastrointestinal syndrome in mice. PLoS One (2009) 4:e8014. doi:10.1371/journal.pone.0008014

67. Zhou WJ, Geng ZH, Spence JR, Geng JG. Induction of intestinal stem cells by R-spondin 1 and Slit2 augments chemoradioprotection. Nature (2013) 501:107-11. doi:10.1038/nature12416

68. Wang B, Xiao Y, Ding BB, Zhang N, Yuan X, Gui L, et al. Induction of tumor angiogenesis by Slit-Robo signaling and inhibition of cancer growth by blocking Robo activity. Cancer Cell (2003) 4:19-29. doi:10.1016/S1535-6108(03)00164-8

69. Phutthaphadoong S, Yamada Y, Hirata A, Tomita H, Hara A, Limtrakul P, et al. Chemopreventive effect of fermented brown rice and rice bran (FBRA) on the inflammation-related colorectal carcinogenesis in ApcMin/+ mice. Oncol Rep (2010) 23:53-9. doi:10.3892/or_00000605
70. Seshagiri S, Stawiski EW, Durinck S, Modrusan Z, Storm EE, Conboy CB, et al. Recurrent R-spondin fusions in colon cancer. Nature (2012) 488:660-4. doi:10.1038/nature11282

71. Ozsolak F, Milos PM. RNA sequencing: advances, challenges and opportunities. Nat Rev Genet (2011) 12:87-98. doi:10.1038/nrg2934

72. Starr TK, Allaei R, Silverstein KA, Staggs RA, Sarver AL, Bergemann TL, et al. A transposon-based genetic screen in mice identifies genes altered in colorectal cancer. Science (2009) 323:1747-50. doi:10.1126/science.1163040

73. Butler AE, Janson J, Bonner-Weir S, Ritzel R, Rizza RA, Butler PC. Betacell deficit and increased beta-cell apoptosis in humans with type 2 diabetes. Diabetes (2003) 52:102-10. doi:10.2337/diabetes.52.1.102

74. Wong VS, Yeung A, Schultz W, Brubaker PL. R-spondin-1 is a novel beta-cell growth factor and insulin secretagogue. J Biol Chem (2010) 285:21292-302. doi:10.1074/jbc.M110.129874

Conflict of Interest Statement: The authors declare that the research was conducted in the absence of any commercial or financial relationships that could be construed as a potential conflict of interest.

Copyright (c) 2015 Li, Zhang and Mulholland. This is an open-access article distributed under the terms of the Creative Commons Attribution License (CC BY). The use, distribution or reproduction in other forums is permitted, provided the original author(s) or licensor are credited and that the original publication in this journal is cited, in accordance with accepted academic practice. No use, distribution or reproduction is permitted which does not comply with these terms. 